\title{
ON THE NEAR EQUICONTINUITY OF TRANSFORMATION GROUPS
}

\author{
WILLIAM J. GRAY AND FRED A. ROBERSON
}

Let $X$ be a compact metric space and $h$ an auto-homeomorphism of $X$. It was proved in [2] that if $h$ has equicontinuous powers at every $x \in X$ except at a finite set $A$, and if $X-A$ is connected, then the number of points of $A$ is at most two, and each point of $A$ is fixed under $h$. Now let $X$ be a locally connected, locally compact metric space. Kaul [4] proved that if $h$ has equicontinuous powers at every $x \in X$, except at a compact 0 -dimensional subset $A$, and if $X-A$ is connected, then the number of points of $A$ is at most two, and each point of $A$ is fixed under $h$. Here we study the above theorem of Kaul in the setting of transformation groups. After proving a rather general theorem, we will generalize Kaul's theorem (Corollary 3).

If $X$ is a metric space, $d$ is any metric compatible with the topology of $X, x \in X$, and $\epsilon>0$, we define

$$
B(x, \epsilon)=\{y \in X: d(x, y)<\epsilon\} .
$$

If $A \subset X, \operatorname{Fr}(A)$ denotes the boundary of $A$. Let $(X, T, \pi)$ be a transformation group. We say that $T$ is equicontinuous at $x \in X$ if for each $\epsilon>0$ there is a $\delta>0$ such that $[d(x, y)<\delta$ implies $d(x t, y t)<\epsilon]$ for every $t \in T$. A subset $A$ of $X$ is a minimal set if

$$
A \neq \varnothing \quad \text { and } \quad A=\overline{x T} \quad \text { for every } x \in A .
$$

Our reference for dimension theory is the book [3].

THEOREM. Let $(X, T, \pi)$ be a transformation group, where $X$ is a locally connected, locally compact metric space. Let $E(T)$ denote the set of points at which $T$ is equicontinuous and $N(T)=X-E(T)$. Let $E(T)$ be connected and $N(T)$ be compact and zero dimensional. Then $N(T)$ contains at most two minimal sets.

(Note: Since $N(T)$ is compact, nonempty, and $T$-invariant, by [ $\mathbf{1}$, 2.22, p. 15], $N(T)$ contains at least one minimal set.)

Proof. Let $s=\left\{s_{n}: n \in Z^{+}\right\}$be a fixed sequence in $T$. If $x \in X$, let $C(x, s)$ denote the set of cluster points of the sequence $\left\{x s_{n}\right\}$. We define

$$
A=\{x \in E(T): C(x, s) \cap E(T) \neq \varnothing\} .
$$

Received by the editors November 25, 1968. 
We will prove

(1) $A$ is open and closed in $E(T)$.

Choose $z \in A$ and let $x \in C(z, s) \cap E(T)$. Choose $\epsilon>0$ such that

$$
\overline{B(x, \epsilon)} \cap N(T)=\varnothing \quad \text { and } \overline{B(x, \epsilon)}
$$

is compact. Choose $\delta>0$ such that $d(z, y)<\delta$ implies $d(z t, y t)<\epsilon / 2$ for every $t \in T$, and $B(z, \delta) \subset E(T)$. Since $x \in C(z, s)$, we may select a subsequence $u$ of $s$ such that $d\left(x, z u_{n}\right)<\epsilon / 2$ for every $n$. If $y \in B(z, \delta)$, then

$$
d\left(x, y u_{n}\right) \leqq d\left(x, z u_{n}\right)+d\left(z u_{n}, y u_{n}\right)<\epsilon
$$

for every $n$. Thus

$$
y u_{n} \in \overline{B(x, \epsilon)}
$$

for every $n$, hence the sequence $\left\{y u_{n}\right\}$ has a cluster point

$$
w \in \overline{B(x, \epsilon)} \text {. }
$$

Then $w \in C(y, s) \cap E(T)$ and $B(z, \delta) \subset A$. We have shown that $A$ is open in $E(T)$.

We now prove that $A$ is closed in $E(T)$. Let $x \in \bar{A} \cap E(T)$, and choose $\epsilon>0$ such that

$$
\overline{B(x, \epsilon)} \cap N(T)=\varnothing \text { and } \overline{B(x, \epsilon)}
$$

is compact. Choose $\delta>0$ such that $d(x, w)<\delta$ implies $[d(x t, w t)<\epsilon / 3]$ for every $t \in T$. Since $x \in \bar{A}$, we may choose $y \in B(x, \delta) \cap A, z \in C(y, s)$ $\cap E(T)$, and $\delta_{1}>0$ such that $d(z, w)<\delta_{1}$ implies $d(z t, w t)<\epsilon / 6$ for every $t \in T$. Choose a subsequence $u$ of $s$ such that $d\left(z, y u_{n}\right)<\delta_{1}$ for every $n$. Then

$$
d\left(y, y u_{n} u_{1}^{-1}\right) \leqq d\left(y, z u_{1}^{-1}\right)+d\left(z u_{1}^{-1}, y u_{n} u_{1}^{-1}\right)<\epsilon / 3
$$

for every $n$. Thus

$$
d\left(x, x u_{n} u_{1}^{-1}\right) \leqq d(x, y)+d\left(y, y u_{n} u_{1}^{-1}\right)+d\left(y u_{n} u_{1}^{-1}, x u_{n} u_{1}^{-1}\right)<\epsilon
$$

for every $n$, hence

$$
x u_{n} u_{1}^{-1} \in \overline{B(x, \epsilon)} \quad \text { for every } n .
$$

Hence there is a subsequence $\left\{u_{n_{k}}\right\}$ of $u$ such that

$$
\left\{x u_{n_{k}} u_{1}^{-1}\right\} \rightarrow w \in \overline{B(x, \epsilon)} .
$$

Then $\left\{x u_{n_{k}}\right\} \rightarrow w u_{1}$, and since $E(T)$ is invariant under $T$, we have

$$
w u_{1} \in C(x, s) \cap E(T)
$$


so that $x \in A$. We have now shown that $A$ is closed in $E(T)$.

Now let $z \in N(T)$. Since $T$ is not equicontinuous at $z$, there is an $\epsilon>0$ such that for every $\delta>0$, diam $B(z, \delta) t>\epsilon$ for some $t \in T$. Choose a finite open cover $U_{1}, \cdots, U_{n}$ of $N(T)$ such that the closures of the $U_{i}$ are compact and pairwise disjoint, diam $U_{i}<\epsilon$, and $N(T) \cap \operatorname{Fr}\left(U_{i}\right)$ $=\varnothing$ for every $i$. Let $\left\{V_{k} ; k \in Z^{+}\right\}$be a sequence of connected open neighborhoods of $z$ such that diam $V_{k}<1 / k$ and let $U^{\prime}=U_{1} \cup \ldots$ $\cup U_{n}$. For each $k$ there is a $t_{k} \in T$ such that diam $V_{k} t_{k}^{-1}>\epsilon$; we note that $V_{k} t_{k}^{-1}$ meets some $U_{i}$ but can be contained in no $U_{i}$. Since $V_{k} t_{k}^{-1}$ is connected, there is an $x_{k} \in V_{k}$ such that for some $i, x_{k} t_{k}^{-1}$ $\in \operatorname{Fr}\left(U^{\prime}\right)$. Since $\operatorname{Fr}\left(U^{\prime}\right)$ is compact, we may assume that $\left\{x_{k} t_{\boldsymbol{k}}^{-1}\right\}$ $\rightarrow x \in \operatorname{Fr}\left(U^{\prime}\right)$. We prove that $\left\{x t_{k}\right\} \rightarrow z$. Let $\delta>0$ be given. Since $x \in E(T)$, there is a $\delta_{1}>0$ such that $d(x, y)<\delta_{1}$ implies $d(x t, y t)<\delta / 2$ for every $t \in T$; choose $m$ so large that $\left[d\left(x_{k} t_{k}^{-1}, x\right)<\delta_{1}\right]$ and $1 / k<\delta / 2$ for every $k \geqq m$. Then if $k \geqq m$ we have

$$
d\left(z, x t_{k}\right) \leqq d\left(z, x_{k}\right)+d\left(x_{k}, x t_{k}\right)<\delta .
$$

Now if we let $t=\left\{t_{k}\right\}$ we get from (1) that

(2) $C(x, t) \cap E(T)=\varnothing$ for every $x \in E(T)$.

Now let $U$ be an open neighborhood of $z$ such that $\bar{U}$ is compact and $N(T) \cap \operatorname{Fr}(U)=\varnothing$. Let

$$
B=\left\{x \in E(T):\left\{x t_{n}\right\} \text { is eventually in } U\right\} .
$$

We will prove that $B$ is open and closed in $E(T)$.

Let $x \in \bar{B} \cap E(T)$ and assume that $x \in B$. Then there is a subsequence $u$ of $t$ such that $x u_{n} \notin U$ for every $n$. Since $T$ is equicontinuous at $x$, there is an $x_{k} \in B$ such that $d\left(x_{k} u_{n}, x u_{n}\right)<1 / k$ for every $n$. Since $\left\{x_{k} u_{n}\right\}$ is eventually in $U$ we may choose a subsequence $\left\{u_{n_{k}}\right\}$ of $u$ such that

(3) $d\left(x_{k} u_{n_{k}}, x u_{n_{k}}\right)<1 / k$ for every $k$ and $x_{k} u_{n k} \in U$. Then $\left\{x_{k} u_{n_{k}}\right\}$ clusters at a point $y \in \bar{U}$. By (3), $y \in C(x, u)$, hence $y \in \operatorname{Fr}(U)$. Therefore we have $y \in \operatorname{Fr}(U) \cap C(x, t)$, and this contradicts (2). We have shown that $B$ is closed in $E(T)$.

Let $x \in B$ and assume $x \notin \operatorname{int}(B)$. Then for each $k$ choose an open neighborhood $U_{k} \subset E(T)$ of $x$ such that $y \in U_{k}$ implies $d(x s, y s)<1 / k$ for every $s \in T$. Since $U_{k}-B \neq \varnothing$, there is a $y_{k} \in U_{k}$ such that $y_{k} t_{n} \in U$ for infinitely many $n$. Since $x \in B$, we may select a subsequence $u$ of $t$ such that $y_{k} u_{k} \notin U$ and $x u_{k} \in U$ for every $k$. Then $\left\{x u_{k}\right\}$ clusters at a point $[w \in \bar{U}]$. Since $d\left(x u_{k}, y_{k} u_{k}\right)<1 / k$ for every $k,\left\{y_{k} u_{k}\right\}$ clusters at $w$ also. Thus $w \in \operatorname{Fr}(U)$ and hence $C(x, t) \cap E(T) \neq \varnothing$; this contradicts (2), and shows that $B$ is open. Finally, since $B \neq \varnothing$, we find that $B=E(T)$. Since each point of $N(T)$ has a neighborhood base con- 
sisting of open sets with compact closures whose boundaries do not meet $N(T)$, we have shown that

(4) If $z \in N(T)$, there exists a sequence $t$ in $T$ such that $\left\{x t_{n}\right\} \rightarrow z$ for each $x \in E(T)$.

We now show that $N(T)$ contains at most two minimal sets. Assume that $x_{1}, x_{2}$, and $x_{3} \in N(T)$ are such that

$$
\overline{x_{1} T}, \overline{x_{2}} T, \text { and } \overline{x_{3} T}
$$

are distinct and minimal. Since minimal sets are disjoint we must have

$$
\overline{x_{1} T} \cap \overline{x_{2}} T=\overline{x_{1} T} \cap \overline{x_{3} T}=\overline{x_{2} T} \cap \overline{x_{3}} T=\varnothing .
$$

Since $N(T)$ is compact and zero dimensional and $X$ is locally compact, we may choose an open subset $W$ of $X$ such that

$$
\overline{x_{1} T} \subset W, \quad \bar{W} \text { is compact, } \bar{W} \cap\left(\overline{x_{2} T} \cup \overline{x_{3} T}\right)=\varnothing,
$$

and $N(T) \cap \operatorname{Fr}(W)=\varnothing$. Choose connected open neighborhoods $V$ and $U$ of $x_{1}$ and $x_{2}$, respectively, such that $V \subset W, \bar{U}$ is compact, and

$$
\bar{U} \cap \overline{x_{3} T}=\varnothing .
$$

Since $E(T)$ is dense in $X$, we may select $y \in U \cap E(T)$. By (4), there is an $s_{1} \in T$ such that $y s_{1} \in V$. Then $C=\bar{V} \cup \bar{U} s_{1}$ is a compact connected set such that

$$
C \cap \overline{x_{1} T} \neq \varnothing, \quad C \cap \overline{x_{2} T} \neq \varnothing,
$$

and

$$
C \cap \overline{x_{3} T}=\varnothing .
$$

Now choose an open neighborhood $M$ of $x_{3}$ such that the closure of $M$ does not meet the set $C$. Choose an $\epsilon>0$ such that $\bar{M} \cap B(C, \epsilon)=\varnothing$, and let $s$ be any sequence in $T$. Then for each integer $n$ we have that

$$
\overline{x_{1}} T \cap C s_{n}^{-1} \neq \varnothing
$$

and

$$
\overline{x_{2} T} \cap C s_{n}^{-1} \neq \varnothing,
$$

and since $C s_{n}^{-1}$ is connected, we must have $C s_{n}^{-1} \cap \operatorname{Fr}(W) \neq \varnothing$. Thus for each $n$, we have $y_{n} \in C$ such that $y_{n} s_{n}^{-1} \in \operatorname{Fr}(W)$. Since $\operatorname{Fr}(W)$ and $C$ are compact, there is a subsequence $\left\{s_{n_{k}}\right\}$ of $s$ such that $\left\{y_{n_{k}} s_{n_{k}}^{-1}\right\}$ $\rightarrow y \in \operatorname{Fr}(W)$ and $\left\{y_{n_{k}}\right\} \rightarrow x \in C$. Because $y \in E(T)$, we may select $\delta>0$ such that if $d(w, y)<\delta$ then $d(w u, y u)<\epsilon / 2$, all $u \in T$. Choose $m$ so large that $k \geqq m$ implies $d\left(y, y_{n_{k}} s_{n_{k}}^{-1}\right)<\delta$ and $d\left(y_{n_{k}}, x\right)<\epsilon / 2$. Then if 
$k \geqq m$, we have

$$
d\left(x, y s_{n_{k}}\right) \leqq d\left(x, y_{n_{k}}\right)+d\left(y_{n_{k}}, y s_{n_{k}}\right)<\epsilon .
$$

This means $y s_{n_{k}} \in B(C, \epsilon)$ for every $k \geqq m$. Thus the sequence $\left\{y s_{n}\right\}$ cannot eventually be in $M$. Since $s$ is an arbitrary sequence in $T$, this contradicts (4) and completes the proof.

In the following corollaries the hypothesis of the theorem is assumed.

Corollary 1. If $x_{1}, x_{2}$, and $x_{3} \in N(T)$, then of the three orbit closures $\overline{x_{1} T}, \quad \overline{x_{2} T}$, and $\overline{x_{3} T}$,

at least two have a nonempty intersection.

Proof. Each $\overline{x_{i} T}$ contains a minimal set $[1,2.22$, p. 15]. The result follows from the theorem.

Corollary 2. If $T$ is almost periodic at each point of $N(T)$, then $N(T)$ is either minimal or is the union of two minimal sets.

Proof. By $[1,4.07$, p. 31$]$, each orbit closure of a point in $N(T)$ is minimal. The result follows from the theorem.

CoROLlaRy 3. If $T$ is abelian or connected, then each point of $N(T)$ is fixed under $T$ and $N(T)$ contains at most two points.

Proof. If $T$ is connected, the orbit of each point of $N(T)$ is a connected subset of $N(T)$, hence is a point. If $T$ is abelian, let $z \in N(T)$ and assume there is an $s \in T$ such that $z \neq z s$. Then there is an open neighborhood $U$ of $z$ such that $U$ is compact, $N(T) \cap \operatorname{Fr}(U)=\varnothing$, and $U \cap U s=\varnothing$. By (4), there is a sequence $t$ in $T$ such that $\left\{x t_{n}\right\}$ is eventually in $U$ for every $x \in E(T)$. Then $\left\{x t_{n}\right\}$ and $\left\{x s^{-1} t_{n}\right\}$ are eventually in $U$, hence there is an integer $m$ such that $x t_{m}, x s^{-1} t_{m} \in U$. But this means that $x t_{m} \in U \cap U s$, and contradicts the choice of $U$. Therefore $\boldsymbol{z}$ is fixed under $T$.

We have shown that $T$ leaves every point of $N(T)$ fixed. Thus each point of $N(T)$ is a minimal set, and the corollary follows from the theorem.

\section{REFERENCES}

1. W. H. Gottschalk and G. A. Hedlund, Topological dynamics, Amer. Math. Soc. Colloq. Publ. Vol. 36, Amer. Math. Soc., Providence, R. I., 1955.

2. T. Homma and S. Kinoshita, On homeomorphisms which are regular except for a finite number of points, Osaka J. Math. 7 (1955), 29-38.

3. J. Nagata, Modern dimension theory, Interscience, New York, 1965.

4. S. K. Kaul, On almost regular homeomorphisms, Canad. J. Math. 20 (1968), 1-6. 